\title{
Magnetic fields generated by r-modes in accreting millisecond pulsars
}

\author{
Carmine Cuofand* and Alessandro Drago \\ Dipartimento di Fisica, Universitá di Ferrara and INFN sezione di Ferrara, 44100 Ferrara, Italy
}

\begin{abstract}
In rotating neutron stars the existence of the Coriolis force allows the presence of the so-called Rossby oscillations (r-modes) which are known to be unstable to emission of gravitational waves. Here, for the first time, we introduce the magnetic damping rate in the evolution equations of r-modes. We show that r-modes can generate very strong toroidal fields in the core of accreting millisecond pulsars by inducing differential rotation. We shortly discuss the instabilities of the generated magnetic field and its long time-scale evolution in order to clarify how the generated magnetic field can stabilize the star.
\end{abstract}

PACS numbers: 04.40.Dg, 04.30.Tv, 04.30.Db, 97.60.Jd

\section{INTRODUCTION}

R-mode oscillations are present in all rotating stars and they are unstable to emission of gravitational waves [1]. These modes play therefore a very important role in the astrophysics of compact stars and in the search for gravitational waves. R-mode instabilities are associated with kinematical secular effects which generate differential rotation in the star and large scale mass drifts, particularly in the azimuthal direction. Differential rotation in turn can produce very strong toroidal magnetic fields inside the star and these fields damp instabilities converting the energy of the mode into magnetic energy. This mechanism has been proposed in the case of rapidly rotating, isolated and newly born neutron stars in Refs. [2, 3]. In our work we consider the back-reaction of the magnetic fields on r-mode instabilities by inserting for the first time the magnetic damping rate into the evolution equations of r-modes. In this way we can follow the temporal evolution of both magnetic fields and r-modes even on a long time scale. In particular we show that taking into account the r-mode instabilities it is possible to generate very strong magnetic fields in accreting millisecond pulsars.

It is important to recall that r-mode instabilities are damped also by the viscosity of the system, either shear or bulk [1]. At low temperature a very important role could also be played by the so-called Ekman layer, located at the interface between the solid crust and the fluid inner core. The friction in the Ekman layer can be significantly enhanced with respect to friction in a purely fluid component. Anyway it is very difficult to give a precise estimate of this effect [4, 5], and in our analysis we have not included the Ekman layer in order to study in a more clear way the effects of magnetic fields on r-modes. We will anyway shortly discuss how the presence of the Ekman layer can modify our results.

The paper is organized as follows: in Sec. II we write the r-mode equations taking into account also the magnetic

*Electronic address: cuofano@fe.infn.it damping rate; in Sec. III we discuss the equations regulating the time evolution of the magnetic field and of the damping rate; in Sec. IV we discuss numerical solutions of the equations; in Sec. $\mathrm{V}$ we discuss open problems concerning the possible presence of the Ekman layer, the effect of a superconducting shell and instabilities of the generated internal magnetic field. Finally in Sec. VI we drive our conclusions.

\section{R-MODE EQUATIONS}

We want to derive the equations regulating the evolution of r-modes in the presence of a pre-existent poloidal magnetic field $B_{p}$ and of the generated internal field. We start by considering the conservation of angular momentum, following Ref. [6]. The total angular momentum $J$ of a star can be decomposed into an equilibrium angular momentum $J_{*}$ and a canonical angular momentum $J_{c}$ proportional to the r-mode perturbation:

$$
J=J_{*}(M, \Omega)+\left(1-K_{j}\right) J_{c}, \quad J_{c}=-K_{c} \alpha^{2} J_{*}
$$

where $K_{(j, c)}$ are dimensionless constants and $J_{*} \cong I_{*} \Omega$. Following Ref. [7] the canonical angular momentum obeys the following equation:

$$
\begin{aligned}
d J_{c} / d t= & 2 J_{c}\left\{F_{g}(M, \Omega)\right. \\
& \left.-\left[F_{v}\left(M, \Omega, T_{v}\right)+F_{m_{i}}(M, \Omega, B)\right]\right\}
\end{aligned}
$$

where $F_{g}$ is the gravitational radiation growth rate, $F_{v}=$ $F_{s}+F_{b}$ is the sum of the shear and bulk viscous damping rate, $T_{v}(t)$ is a spatially averaged temperature and now we also have introduced the damping rate $F_{m_{i}}$, associated with the generated internal magnetic field. The explicit expression of $F_{m_{i}}$ will be discussed in the following. The total angular momentum satisfies the equation:

$$
d J / d t=2 J_{c} F_{g}+\dot{J}_{a}(t)-I_{*} \Omega F_{m_{e}}
$$

where $\dot{J}_{a}$ is the rate of accretion of angular momentum (we have assumed it to be $\dot{J}_{a}=\dot{M}(G M R)^{1 / 2}$, see Ref. [8]) and $F_{m_{e}}$ is the magnetic breaking rate associated to the external poloidal magnetic field. In the present paper we are not considering further breaking mechanisms 

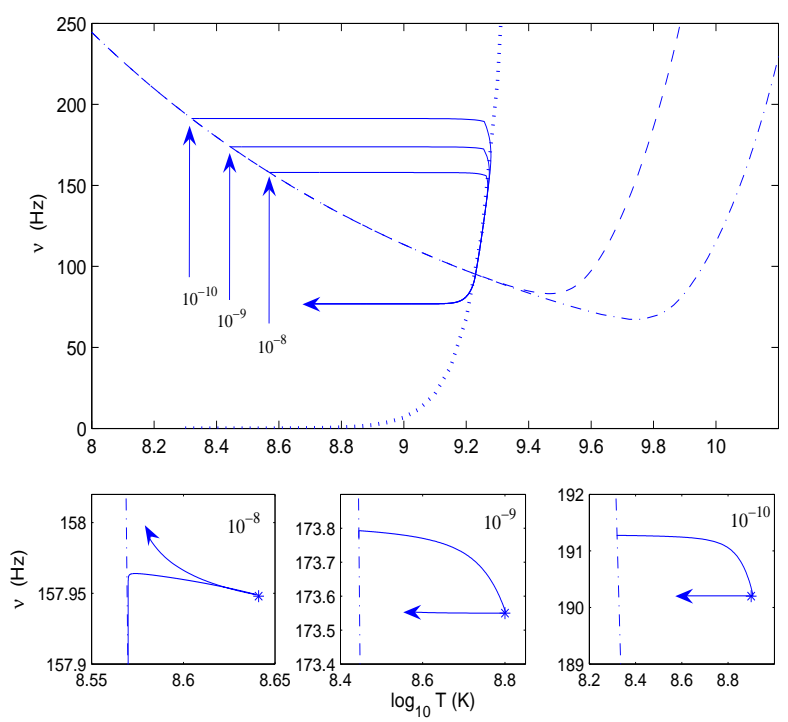

FIG. 1: Top panel: instability region in the Temperature vs Frequency plane obtained using either the bulk viscosity damping rate $F_{b}$ given in [1] (region above the dotdashed line) or the $F_{b}$ given in [9] (region above the dashed line). Also shown are the paths followed by accreting neutron stars, without toroidal magnetic fields (solid lines). The three curves correspond to different values of accretion rate $\dot{M}=\left(10^{-8}, 10^{-9}, 10^{-10}\right) \mathrm{M}_{\odot} \mathrm{yr}^{-1}$. We plot also the temperature equilibrium curve [5] (dotted line), obtained taking into account the neutrino cooling and the reheating due to viscosity. Bottom panels: new paths obtained taking into account the new generated toroidal fields. Here $B_{d}=10^{8} \mathrm{G}$. The moments at which toroidal magnetic fields damp r-mode instabilities are indicated by asterisks.

as e.g. the interaction between the magnetic field and the accretion disk. Combining Eqs. (2) and (3) we obtain the evolution equations of the r-mode amplitude $\alpha$ and of the angular velocity of the star $\Omega$ :

$$
\begin{aligned}
\frac{d \alpha}{d t}= & \alpha\left(F_{g}-F_{v}-F_{m_{i}}\right) \\
& +\alpha\left[K_{j} F_{g}+\left(1-K_{j}\right)\left(F_{v}+F_{m_{i}}\right)\right] K_{c} \alpha^{2} \\
& -\frac{\alpha \dot{M}}{2 \tilde{I} \Omega}\left(\frac{G}{M R^{3}}\right)^{1 / 2}+\frac{\alpha F_{m_{e}}}{2} \\
\frac{d \Omega}{d t}=\quad & -2 K_{c} \Omega \alpha^{2}\left[K_{j} F_{g}+\left(1-K_{j}\right)\left(F_{v}+F_{m_{i}}\right)\right] \\
& -\frac{\dot{M} \Omega}{M}+\frac{\dot{M}}{\tilde{I}}\left(\frac{G}{M R^{3}}\right)^{1 / 2}-\Omega F_{m_{e}}
\end{aligned}
$$

where $I_{*}=\tilde{I} M R^{2}$ with $\tilde{I}=0.261$ for an $\mathrm{n}=1$ polytrope and $K_{c}=9.4 \times 10^{-2}$, see Ref. [9]. Our results turn out to be rather insensitive to the value of $K_{j} \sim 1$ (see Ref. [6]).

\section{MAGNETIC DAMPING}

The crucial ingredient introduced before is the magnetic damping rate $F_{m_{i}}$, which we have inserted in the evolution of r-modes. The expression of the magnetic damping rate has been derived in [2, 3], where it has been shown that while the star remains in the instability region, the r-modes generate a differential rotation which can greatly amplify a pre-existent magnetic field. More specifically, if a poloidal magnetic field was originally present, a strong toroidal field is generated inside the star. The energy of the modes is therefore transferred to the magnetic field and the instability is damped.

We assume that the stellar magnetic field $\mathbf{B}$ is initially dipolar and aligned with the star's spin axis

$$
\mathbf{B}_{0}=\mathbf{B}^{p}(t=0)=B_{d} \frac{R^{3}}{r^{3}}\left(2 \cos \theta \mathbf{e}_{r}+\sin \theta \mathbf{e}_{\theta}\right)
$$

where $B_{d}$ is the strength of the equatorial magnetic field at the stellar surface. The initial magnetic field is assumed to be described by Eq. (6) both inside and outside. In order to avoid divergences we assume the previous radial dependence of the dipolar field to hold only for $r \geq R / 2$. If for instance the dipolar field remains roughly constant in the inner region its contribution to the total energy of the final toroidal field is negligible. Therefore in the following and in agreement with Ref. 3], radial integrations extend from $R / 2$ to $R$.

To estimate the magnetic field produced by r-modes we start by writing the $l=m=2$ contribution to the perturbation velocity:

$$
\delta \mathbf{v}(r, \theta, \phi, t)=\alpha \Omega R\left(\frac{r}{R}\right)^{2} \mathbf{Y}_{22}^{B} e^{i \sigma t}
$$

Following Ref. [2] we get the total azimuthal displacement from the onset of the oscillation at $t_{0}$ up to time $t$, which reads:

$$
\begin{aligned}
& \Delta \tilde{x}^{\phi}(r, t) \equiv \int_{t_{0}}^{t} \delta v^{\phi}\left(t^{\prime}\right) d t^{\prime} \\
& =\frac{2}{3}\left(\frac{r}{R}\right) k_{2}(\theta) \int_{t_{0}}^{t} \alpha^{2}\left(t^{\prime}\right) \Omega\left(t^{\prime}\right) d t^{\prime}+\mathcal{O}\left(\alpha^{3}\right)
\end{aligned}
$$

where $k_{2}(\theta) \equiv(1 / 2)^{7}(5 ! / \pi)\left(\sin ^{2} \theta-2 \cos ^{2} \theta\right)$. The relation between the new and the original magnetic field inside the star in the Lagrangian approach reads [2]:

$$
\frac{B^{j}}{\rho}(\tilde{\mathbf{x}}, t)=\frac{B^{k}}{\rho}\left(\mathbf{x}, t_{0}\right) \frac{\partial \tilde{x_{j}}(t)}{\partial x^{k}\left(t_{0}\right)} .
$$

This equation implies that the radial dependence of the initial and final magnetic field is the same. Integrating on time the induction equation in the Eulerian approach one gets [3]:

$$
\begin{aligned}
\delta B^{\theta} & \simeq \delta B^{r} \simeq 0 \\
\delta B^{\phi} & \simeq B_{0}^{\theta} \int \dot{\phi}\left(t^{\prime}\right) d t^{\prime} \simeq B_{0}^{\theta} \int \frac{\delta v^{\phi}\left(t^{\prime}\right)}{r} d t^{\prime}
\end{aligned}
$$


where $B_{\phi}$ is the toroidal component.

The expression of the magnetic damping rate reads:

$$
\begin{aligned}
F_{m_{i}}(t) & =\frac{\left(d E_{M} / d t\right)}{\tilde{E}} \\
\simeq & \frac{4(1-p)}{9 \pi p \cdot\left(8.2 \times 10^{-3}\right)} \frac{B_{p}^{2} R \Lambda^{\prime} \int_{0}^{t} \alpha^{2}\left(t^{\prime}\right) \Omega\left(t^{\prime}\right) d t^{\prime}}{M \Omega}
\end{aligned}
$$

where $\tilde{E}$ is the energy of the mode, $E_{M}$ is the magnetic energy, $\Lambda^{\prime} \approx \mathcal{O}(1)$ is a dimensionless parameter [3] and $p=0.5$ indicates that we are integrating from $R / 2$ to $R$. The time integral over the r-mode amplitude $\alpha$ takes contribution from the period during which the star is inside the instability region.

\section{NUMERICAL SOLUTIONS}

In our numerical analysis we use the estimate given in [1] for the gravitational radiation reaction rate due to the $l=m=2$ current multipole

$$
F_{g}=\frac{1}{47} M_{1.4} R_{10}^{4} P_{-3}^{-6} \quad \mathrm{~s}^{-1}
$$

as well as for the bulk and shear viscosity damping rates

$$
\begin{aligned}
F_{b} & =\frac{1}{2.7 \times 10^{11}} M_{1.4}^{-1} R_{10} P_{-3}^{-2} T_{9}^{6} \mathrm{~s}^{-1} \\
F_{s} & =\frac{1}{2.2 \times 10^{7}} M_{1.4} R_{10}^{-5} T_{9}^{-2} \mathrm{~s}^{-1}
\end{aligned}
$$

where we have used the notation $M_{1.4}=M / 1.4 M_{\odot}$, $R_{10}=R / 10 \mathrm{Km}, P_{-3}=P / 1 \mathrm{~ms}$ and $T_{9}=T / 10^{9} \mathrm{~K}$. It is important to remark that the damping rate $F_{b}$ depends on the density profile of the star. Using a different profile in [9] they obtained a value for $F_{b}$ three orders of magnitude larger than in [1]. Fortunately our results are insensitive to this quantity, only the instability window is modified as shown in Fig. 1.

Viscosity depends critically on temperature. We include three factors in modeling the temperature evolution: modified Urca cooling, shear viscosity reheating, and accretion heating. The cooling rate due to the modified Urca reactions, $\dot{\epsilon}_{u}$, reads [10]

$$
\dot{\epsilon}_{u}=7.5 \times 10^{39} M_{1.4}^{2 / 3} T_{9}^{8} \quad \operatorname{erg~s}^{-1} .
$$

The neutron star will be heated by the action of shear viscosity on the r-mode oscillations. The heating rate due to shear viscosity, $\dot{\epsilon}_{s}$, reads [1]

$$
\begin{aligned}
\dot{\epsilon}_{s} & =2 \alpha^{2} \Omega^{2} M R^{2} \tilde{J} F_{s} \\
& =8.3 \times 10^{37} \alpha^{2} \Omega^{2} \tilde{J} M_{1.4}^{9 / 4} R_{10}^{-15 / 4} T_{9}^{-2} \quad \operatorname{erg~s}^{-1} .
\end{aligned}
$$

where $\tilde{J}=1.635 \times 10^{-2}$.

Accretion heating has two components. We use the estimates given in [11]. The first contribution arises when
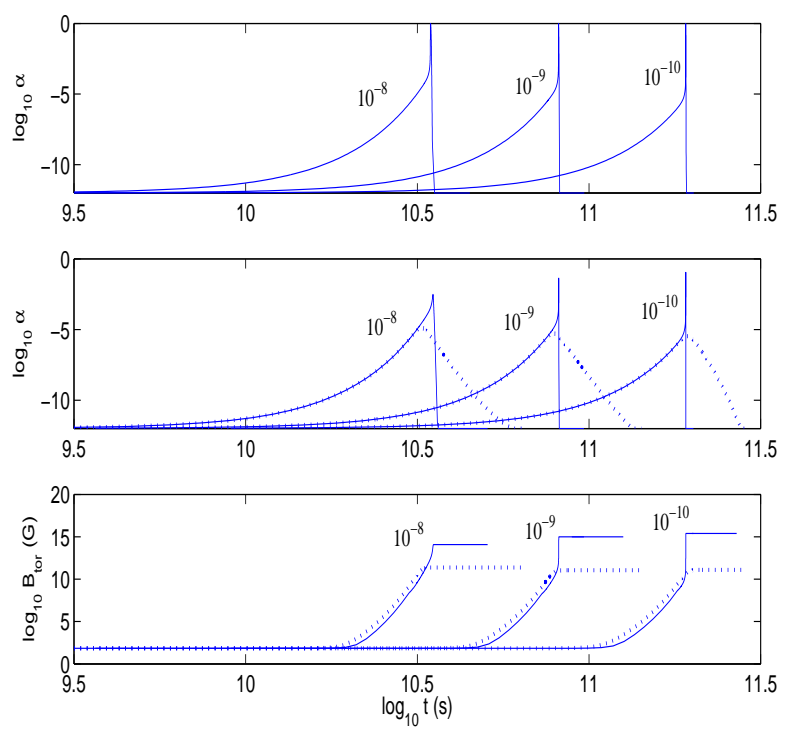

FIG. 2: Top panel: temporal evolution of r-modes amplitude without toroidal fields. Middle panel: temporal evolution of r-modes amplitude with toroidal fields for $B_{d}=10^{8} \mathrm{G}$ (solid line) and $B_{d}=10^{9} \mathrm{G}$ (dotted line). Bottom panel: temporal evolution of volume averaged toroidal magnetic fields. We consider $\dot{M}=\left(10^{-8}, 10^{-9}, 10^{-10}\right) \mathrm{M}_{\odot} \mathrm{yr}^{-1}$.

accreted matter undergoes nuclear burning at the surface of the star

$$
\dot{\epsilon}_{n}=\frac{\dot{M}}{m_{B}} \times 1.5 \mathrm{MeV}=4 \times 10^{51} \dot{M}_{1.4} \quad \mathrm{erg} \mathrm{s}^{-1}
$$

where $m_{B}$ is the mass of a baryon.

The second contribution arises because the flow is assumed to be advection dominated. The heating rate is

$$
\dot{\epsilon}_{h} \sim \frac{R}{\lambda} \frac{G M \dot{M}}{R}=8 \times 10^{51} M_{1.4}^{13 / 6} \dot{M}_{1.4} \quad \operatorname{erg~s}^{-1}
$$

Finally we use the estimate of the heat capacity $C_{v}$ given in [11]

$$
C_{v}=1.6 \times 10^{39} M_{1.4}^{1 / 3} T_{9} \quad \operatorname{erg~K}{ }^{-1} .
$$

The equation of thermal balance of the star is

$$
\frac{d}{d t}\left[\frac{1}{2} C_{v} T\right]=\dot{\epsilon}_{s}-\dot{\epsilon}_{u}+\dot{\epsilon}_{n}+\dot{\epsilon}_{h} .
$$

We consider a scenario in which the mass accretion spins an initially slowly rotating neutron star up to millisecond period and we investigate the evolution of internal toroidal magnetic fields when the star enters the r-modes instability window.

We start by discussing the evolution of temperature and spin frequency obtained without magnetic fields. In 
Fig. 1 we show that the star crosses the r-mode instability region in a regime of thermo-gravitational runaway [5]. R-modes grow exponentially due to the decrease of the shear viscosity with increasing temperature. As a consequence r-mode amplitudes rapidly reach the saturation value (we chose $\alpha_{\text {sat }}=1$ although, as we shall see later, magnetic fields limit $\alpha \ll \alpha_{\text {sat }}$ ) and the viscosity heats significantly the star. At this stage the star loses angular momentum by emission of gravitational waves and goes out of the instability region in a time of hundred of years.

Taking into account magnetic fields, the evolutionary scenario for the star is quite different. We discuss results obtained solving in a self-consistent way Eqs. (4511119). From a practical viewpoint we proceed as follows: we first solve Eqs. (4519) and we get an estimate of $F_{m_{i}}(t)$ inserting the results in Eq. (11); we use this estimate to solve again Eqs. (4519). This procedure is iterated till numerical convergence is reached.

The exponential growth of r-modes induces extremely large secular effects and the toroidal magnetic field is either produced or amplified by the wrapping of the poloidal field produced by the secular velocity field which is mostly toroidal. In the bottom panel of Fig. 1 we show the new trajectory of the star in the TemperatureFrequency plane obtained taking into account the generated toroidal fields, and we indicate with asterisks the moments at which magnetic fields damp r-modes. It is important to remark that this happens when the star is still in the region which was unstable taking into account only the viscous damping.

In Fig. 2 we show the evolution of the r-mode amplitude $\alpha$, without magnetic field (top panel) and with magnetic field (middle panel). Three different values of accretion rate $\dot{M}$ and two values of the initial poloidal magnetic field $B_{d}$ are considered. In the scenario with magnetic fields, the maximum values of $\alpha$ are in the range $\alpha_{\max } \sim\left[10^{-6}-10^{-1}\right]$ and the generated toroidal fields are in the range $B_{\text {tor }} \sim\left[10^{11}-10^{15}\right] \mathrm{G}$ (Fig. 2 bottom panel). In this analysis we have considered values of accretion rate $\dot{M}$ and magnetic field $B_{d}$ typical of accreting Low Mass X-Ray binaries (LMXBs).

In Fig. 3 we show the generated toroidal magnetic field as a function of the temperature at which the star enters the instability window. We display the volume averaged field obtained at the moment in which the field itself has completely damped $\mathrm{r}$-modes. The value of the magnetic field is the asymptotic one displayed in the bottom panel of Fig. 2, Results of Fig. 3are obtained using Eqs. (16 17) to describe the connection between accretion rate $\dot{M}$ and temperature $T$, but they are rather insensitive to the exact relation between these two quantities.

\section{OPEN PROBLEMS}

In our work we have not discussed the possible existence of Ekman layer. If this layer is present it stabilizes

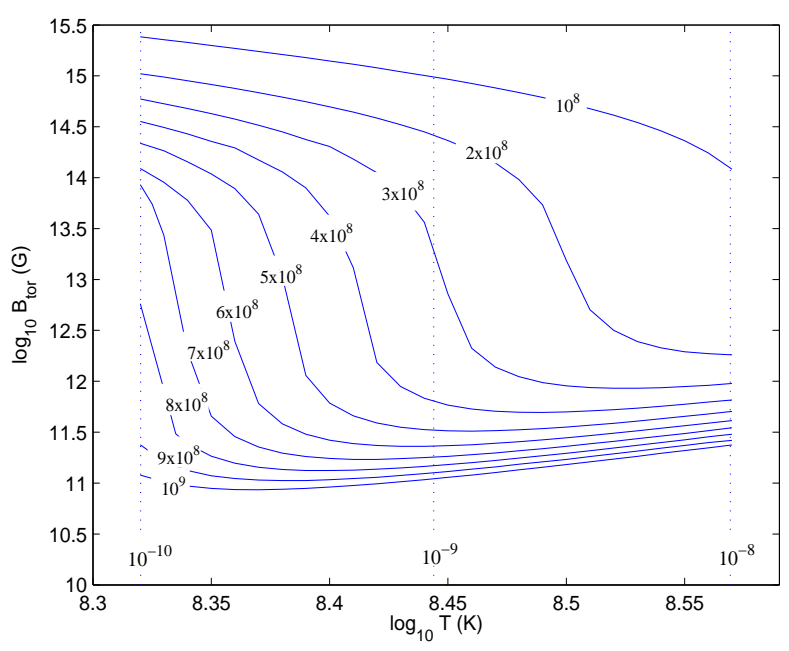

FIG. 3: Volume averaged toroidal magnetic field generated by r-modes as a function of the temperature at which the star enters the instability window. For reference we show also the value of the temperatures which correspond to a mass accretion rate $\dot{M}=\left(10^{-8}, 10^{-9}, 10^{-10}\right) \mathrm{M}_{\odot} \mathrm{yr}^{-1}$. The various curves correspond to different values of the initial poloidal magnetic field, in the range $\left[10^{8}-10^{9}\right] \mathrm{G}$.

the star up to frequencies of a few hundred Hertz. The scenario discussed above does not change qualitatively, but since the star enters the instability region at higher frequencies, the growth rate of magnetic fields is larger. Another important open point concerns the possible formation of superconductivity in the core of neutron stars. A fraction of the core, whose temperature is below the critical value $T_{c} \sim 10^{9} \mathrm{~K}$, is expected to be either a Type I [12, 13] or a Type II superconductor [14, 15]. The exact nature of the possible superconducting layer is still uncertain as well as the precise value of the superconducting gap. The fundamental quantity in our analysis is the size of the superconducting region which we show in Fig. 4using the results obtained in [14]. The thickness of the superconducting shell is about $1 \mathrm{~km}$. In the same figure we also display the energy density of the r-modes. It is clear that most of the volume where r-modes can develop remains not superconducting and in that region our analysis can still be applied: r-modes are damped by the magnetic field and therefore the perturbation velocity drops to zero. R-modes can develop in the thin superconducting layer, but their amplitude is strongly suppressed because the perturbation velocity has to vanish at the interface between the superconducting and the not superconducting region. Moreover, if the superconductivity is of Type II, the magnetic flux of the original poloidal field organizes into quantized flux tubes. R-modes will stretch these flux tubes, increasing their length and thus changing their magnetic energy. As in the case of the normal core, this process can generate very intense magnetic 


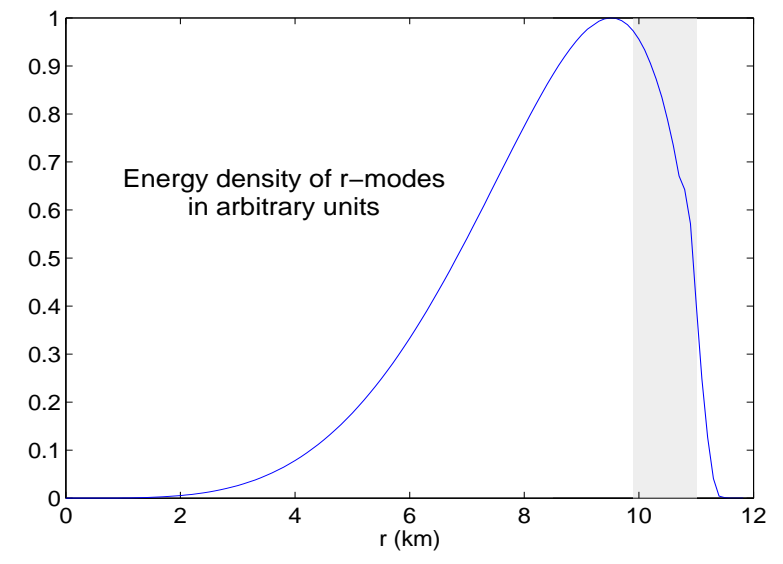

FIG. 4: Energy density of r-modes as a function of the star radius. The estimates about the size of the superconducting region are quite uncertain. Recent results obtained in [14] suggest that the superconducting shell has a thickness $\lesssim 1$ $\mathrm{km}$ and it is located in the grey area.

fields which lead to the damping of the r-mode oscillations even in the superconducting layer [3]

Several issues remain open concerning how the new generated magnetic fields are affected by possible instabilities. In the stably stratified environment of a stellar interior there are two types of instabilities: the Parker (or magnetic buoyancy) and the Tayler instabilities (or pinch-type), both driven by the magnetic field energy in the toroidal field. The buoyancy instability is negligible for $B_{\text {tor }} \lesssim 10^{15} \mathrm{G}$, so we focus on Tayler instabilities because they set in at a lower field strength [16]. It is important to remark that in a stable and stratified neutron star the condition for the Tayler instability reads [17]:

$$
\frac{\omega_{A}}{\Omega}>\left(\frac{N_{\mu}}{\Omega}\right)^{1 / 2}\left(\frac{\eta}{r^{2} \Omega}\right)^{1 / 4}
$$

where $\omega_{A}=B /(4 \pi \rho)^{1 / 2} r$ is the Alfvén frequency, $N_{\mu} \simeq$ $5 \times 10^{4} \mathrm{~s}^{-1}$ is the compositional contribution to the buoyancy frequency and $\eta \sim 10^{-9} \mathrm{~cm}^{2} \mathrm{~s}^{-1}$ is the magnetic diffusivity [18. From Eq. (20) we can conclude that in the stably stratified core of a neutron star, the Tayler instability sets in for $B_{\text {tor }}^{c r} \gtrsim 10^{12} \mathrm{G}$.

After the development of the Tayler instability, the toroidal component of the field produces, as a result of its decay, a new poloidal component which can then be wound up itself, closing the dynamo loop. Both components then grow, more slowly, until the saturation level is reached, when the field is being destroyed by the instability at the same rate at which it is being amplified by the differential rotation [19]. When the differential rotation stops the field can evolve into a stable configuration of a mixed poloidal-toroidal twisted-torus shape embedded inside the star with an approximately dipolar field con-

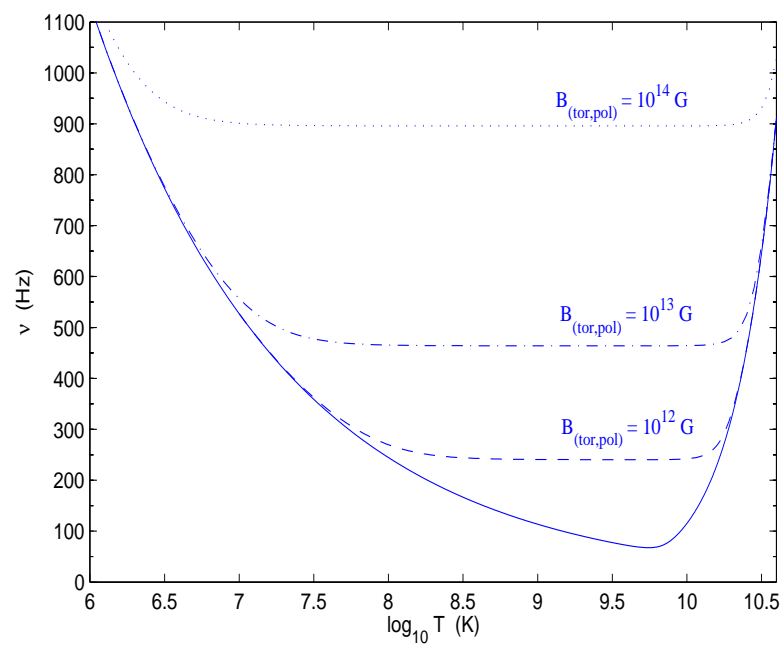

FIG. 5: New r-modes instability regions for neutron stars with stable configurations of mixed poloidal-toroidal fields in the inner core (no magnetic field - solid line; $B_{t o r, p o l}=10^{12} \mathrm{G}$ dashed line; $B_{\text {tor }, \text { pol }}=10^{13} \mathrm{G}$ - dotted-dashed line; $B_{\text {tor }, p o l}=$ $10^{14} \mathrm{G}$ - dotted line). We assume that poloidal and toroidal components have similar strengths.

nected to it outside the star [20, 21, 22, 23]. Once the field is stabilized it should evolve as a result of diffusive processes as Ohmic dissipation, ambipolar diffusion, and Hall drift [24], whose typical time-scales are:

$$
\begin{aligned}
t_{\text {ohmic }} & \sim 2 \times 10^{11} \frac{L_{5}^{2}}{T_{8}^{2}}\left(\frac{\rho}{\rho_{\text {nuc }}}\right)^{3} \mathrm{yr} \\
t_{\text {ambip }} & \sim 3 \times 10^{9} \frac{T_{8}^{2} L_{5}^{2}}{B_{12}^{2}} \mathrm{yr} \\
t_{\text {Hall }} & \sim 5 \times 10^{8} \frac{L_{5}^{2}}{B_{12}}\left(\frac{\rho}{\rho_{\text {nuc }}}\right) \mathrm{yr}
\end{aligned}
$$

where $L_{5}=L / 10^{5} \mathrm{~cm}$ is the size of the region embedding the magnetic field.

\section{CONCLUSIONS}

We have shown how r-modes can generate strong toroidal fields in the core of accreting millisecond neutron stars, and how these fields influence the growth rate of $r$ mode instabilities. Tayler instability sets in for strengths of the generated fields of the order of $10^{12} \mathrm{G}$ and stabilizes the toroidal component by producing a new poloidal field of similar strength. This stable configuration evolves on a time-scale, regulated by diffusive processes. Our results imply that in the core of accreting neutron stars in LMXBs, rotating at frequencies $\nu \gtrsim 200 \mathrm{~Hz}$, there are strong magnetic fields with strengths $B \gtrsim 10^{12} \mathrm{G}$.

Finally, it is tempting to try to investigate how the new 
stable configuration of magnetic fields modifies the instability window of r-modes. More explicitly the scenario we have in mind is the following:

- the compact star inside the LMXB initially enters the instability window and it follows the trajectories that are displayed in the bottom panel of Fig. 1 and in Fig. 2. When the toroidal field reaches the critical value dictated by the Tayler instability, the poloidal field suddenly increases and we assume it reaches a value of the same order of $B_{t o r}^{c r}$. The new magnetic configuration, in which the poloidal and the toroidal field are of the same order of magnitude, is stable. In the new configuration the internal poloidal field is a few orders of magnitude larger than the initial poloidal field. R-modes try again to deform the poloidal field generating a new toroidal component but the magnetic damping rate given by Eq. (11) is now much larger and the star is stable with respect to $\mathrm{r}$-modes up to frequencies of the order of $200 \mathrm{~Hz}$.

- the star continues accreting and increasing its frequency and it enters again the instability region generating again a new toroidal field. When the new toroidal component becomes much larger than the poloidal component Tayler instability sets in again increasing the value of the poloidal field. In Fig. 5 we show how the instability window changes in dependence of the internal magnetic configuration, assuming that the toroidal and poloidal components are equal.

- to determine the actual maximum value of the limiting frequency we take into account also the timescale associated with the diffusive processes described by Eqs. (21) 23). If the magnetic fields exceed a few $10^{14} \mathrm{G}$ the diffusion time-scale becomes shorter than $10^{6} \mathrm{yr}$ and the star cannot accelerate further even for very large values of $\dot{M}$.

It is clear that even in the absence of the Ekman layer the new internal magnetic fields can stabilize stars with frequencies up to several hundreds Hertz. The possible presence of a superconducting shell would screen these internal fields so that they would not affect the dynamics of the external region. The phenomenological implications of this model will be discussed in a forthcoming paper.

It is a pleasure to thank Marcello Baldo, Giuseppe Pagliara and Andreas Reisenegger for many useful and stimulating discussions.
[1] N. Andersson and K. D. Kokkotas, Int. J. Mod. Phys. D10, 381 (2001).

[2] L. Rezzolla, F. K. Lamb, D. Markovic, and S. L. Shapiro, Phys. Rev. D64, 104013 (2001).

[3] L. Rezzolla, F. K. Lamb, D. Markovic, and S. L. Shapiro, Phys. Rev. D64, 104014 (2001).

[4] K. Glampedakis and N. Andersson, Mon. Not. Roy. Astron. Soc. 371, 1311 (2006).

[5] R. Bondarescu, S. A. Teukolsky, and I. Wasserman, Phys. Rev. D76, 064019 (2007), 0704.0799.

[6] R. V. Wagoner, Astrophys. J. 578, L63 (2002).

[7] J. L. Friedman and B. F. Schutz, Astrophys. J. 222, 281 (1978).

[8] N. Andersson, D. I. Jones, and K. D. Kokkotas, Mon. Not. Roy. Astron. Soc. 337, 1224 (2002).

[9] B. J. Owen et al., Phys. Rev. D58, 084020 (1998).

[10] S. L. Shapiro and S. A. Teukolsky, Black holes, white dwarfs, and neutron stars: The physics of compact objects (John Wiley \& sons, New York, 1983).

[11] A. L. Watts and N. Andersson, Mon. Not. Roy. Astron. Soc. 333, 943 (2002).

[12] B. Link, Physical Review Letters 91, 101101 (2003).
[13] K. B. Buckley, M. A. Metlitski, and A. R. Zhitnitsky, Physical Review Letters 92, 151102 (2004).

[14] M. Baldo and H.-J. Schulze, Phys. Rev. C75, 025802 (2007).

[15] I. Wasserman, Mon. Not. Roy. Astron. Soc. 341, 1020 (2003).

[16] H. C. Spruit, Astron. Astrophys. 349, 189 (1999).

[17] H. C. Spruit, Astron. Astrophys. 381, 923 (2002).

[18] P. Haensel, A. Y. Potekhin, and D. G. Yakovlev, Neutron stars 1: Equation of state and structure (Springer, New York, 2007).

[19] J. Braithwaite, Astron. Astrophys. 449, 451 (2006).

[20] A. Reisenegger, Astron. Astrophys. 499, 557 (2009).

[21] J. Braithwaite and H. C. Spruit, Astron. Astrophys. 450, 1097 (2006).

[22] J. Braithwaite and H. C. Spruit, Nature. 431, 819 (2004).

[23] J. Braithwaite and Å. Nordlund, Astron. Astrophys. 450, 1077 (2006).

[24] P. Goldreich and A. Reisenegger, Astrophys. J. 395, 250 (1992). 